Pacific Journal of Mathematics

ON THE NON-MONOTONY OF DIMENSION 


\title{
ON THE NON-MONOTONY OF DIMENSION
}

\author{
Beverly L. Brechner
}

In this paper an example is constructed of a compact Hausdorff space $X$ with covering and large inductive dimensions 0 , but containing subsets $Y_{n}$, with covering dimension $n$, and large inductive dimension at least $n$, each $n, 1 \leqq n \leqq$ $\infty$. This result is an interesting contrast with the recent result of $D$. W. Henderson that there exists an infinite dimensional compact metric space with no $n$-dimensional compact subsets for $1 \leqq n<\infty$. As a corollary of our results, we show that for each $n, 1 \leqq n \leqq \infty$, there exists a (necessarily non-metric) continuum $M_{n}$ of covering dimension $n$, which contains subsets of all positive covering dimensions. Covering dimension is treated in $\S 2$, while large inductive dimension is treated in $\S 4$. In $\S 3$, compactifications of $Y_{n}$ are discussed and it is shown that the covering dimension of $\beta Y_{n}=0$ for $1 \leqq n<\infty$. It is known (see Gillman and Jerison) that for a normal space $N, \operatorname{cov} \operatorname{dim} N=\operatorname{cov} \operatorname{dim} \beta N$.

1. Introduction and construction of examples. For separable metric spaces, cov dim $=$ ind $\operatorname{dim}=$ Ind dim, and each of these is monotone; that is, $A \subseteq B$ implies $\operatorname{dim} A \leqq \operatorname{dim} B$. However, it is wellknown that although ind dim is always monotone [7, appendix], cov dim and Ind dim need not be monotone. In the appendix of [7], it is shown that if $S$ denotes the Tychonoff Plank, and $T$ denotes a particular (non-normal) subset of $S$, then ind $\operatorname{dim} S=\operatorname{cov} \operatorname{dim} S=$ Ind $\operatorname{dim} S=0$, while $\operatorname{cov} \operatorname{dim} T>0$ and $\operatorname{Ind} \operatorname{dim} T>0$. However, cov $\operatorname{dim} T$ and Ind $\operatorname{dim} T$ are not determined in [7]. In this paper, we will prove, among other things, that the set $T$ ( $Y_{1}$ in our notation) satitisfies $\operatorname{cov} \operatorname{dim} T=$ Ind $\operatorname{dim} T=1$.

We establish the following notation.

Let $\Omega_{0}$ denote the first ordinal whose cardinal is infinite.

Let $\Omega_{n}$, for each positive integer $n$, denote the first ordinal greater than $\Omega_{n-1}$, whose cardinality is greater than the cardinality of $\Omega_{n-1}$. (Note that $\Omega_{n}$ is a limit ordinal. See page 100 of [5].)

Let $X_{i}$ be the set of ordinals from 0 to $\Omega_{i}$, inclusive, with the order topology; $X_{i}=\left[0, \Omega_{i}\right]$.

Let $X=\prod_{i=0}^{\infty} X_{i}$.

Let $p=\left(\Omega_{0}, \Omega_{1}, \cdots, \Omega_{i}, \cdots\right) \in X$.

Let $Y_{\infty}=Y=X-\{p\}$.

Let $Z_{n}=\prod_{i=0}^{n} X_{i}$.

Let $p_{n}=\left(\Omega_{0}, \Omega_{1}, \cdots, \Omega_{n}\right) \in Z_{n}$. 
Let $Y_{n}=Z_{n}-\left\{p_{n}\right\}$.

The examples of this paper are $X$ and the $Y_{n}$ 's just constructed.

We note that they are simple generalizations of the Tychonoff Plank. (See p. 132 of [9] or p. 154 of [7].) It is known that for any space $A$, Ind $\operatorname{dim} A=0$ iff $\operatorname{cov} \operatorname{dim} A=0$, and that for a compact Hausdorff space $A$, ind $\operatorname{dim} A=0$ iff Ind $\operatorname{dim} A=0$ iff $\operatorname{cov} \operatorname{dim} A=0$. (See [7, appendix] and [10].) Thus a totally disconnected, compact, Hausdorff space has dimension 0 in all three senses. The spaces $Z_{n}, 1 \leqq$ $n<\infty$, and $X$, constructed above, are totally disconnected, compact, Hausdorff, and hence, 0-dimensional in all three senses.

All spaces are Hausdorff.

We use the following definitions.

The small inductive dimension of a space $A$, ind $A$, is -1 , iff $A=\varnothing$; ind $A \leqq n$ iff for every point $p \in A$ and open set $U$ containing $p$, there is an open set $V$ such that $p \in V \leqq U$ and ind (bdry $V$ ) $n-1$. We say ind $A=n$ iff ind $A \leqq n$ but ind $A \leqq n-1$.

The large inductive dimension of a space $A$, Ind $A$, is -1 iff $A=\varnothing ;$ Ind $A \leqq n$ iff for every closed set $F \leqq A$ and open set $U$ containing $F$, there is an open set $V$ such that $F \subseteq V \subseteq U$ and Ind (bdry $V) \leqq n-1$. We say Ind $A=n$ iff Ind $A \leqq n$ but Ind $A \leqq$ $n-1$.

The covering dimension of a space $A, \operatorname{cov} \operatorname{dim} A$, or $\operatorname{dim} A$, is -1 iff $A$ is empty; $\operatorname{dim} A \leqq n$ iff every finite open cover of $A$ has a finite open refinement such that at most $(n+1)$ elements have a nonempty intersection. We say $\operatorname{dim} A=n$ iff $\operatorname{dim} A \leqq n$ but $\operatorname{dim} A \leqq n-1$.

See [7] and [10] for a further discussion of dimension theory.

An n-dimensional cover of a space is a cover in which some $(n+1)$ elements meet, but no $(n+2)$ elements meet.

An initial segment of ordinals in $X_{n}$ (or $X_{n}-\left\{\Omega_{n}\right\}$ ) is the set of all ordinals less than some $\alpha \in X_{n}$ (or $X_{n}-\left\{\Omega_{n}\right\}$ ).

An end segment of ordinals is the complement of an initial segment.

A cofinal set $S$ in $X_{n}$ means if $\alpha \in\left[0, \Omega_{n}\right)$ then there exists $\beta$ such that $\alpha<\beta<\Omega_{n}$ and $\beta \in S$.

2. The non-monotony of covering dimension. We have seen that $\operatorname{dim} X=0$. In this section we prove that $\operatorname{dim} Y_{n}=n$ for $1 \leqq n \leqq$ $\infty$, and that $X$ contains a copy of each $Y_{n}$. It is clear that $Y_{n}$ is not normal for $n \geqq 1$, since it contains, as a closed subset, the nonnormal subset $T$ of the Tychonoff Plank $S$ discussed in $\S 1$. 
We note that Dowker has given an example of a normal Hausdorff space $N$ with a normal subset $M$ such that $\operatorname{dim} N=\operatorname{Ind} N=0$, but $\operatorname{dim} M=$ Ind $M=1$. See [3] or pages 102-3 of [8].

Lemma 2.1. Let $\mathscr{U}: U_{1}, U_{2}, \cdots, U_{k}$ be a finite open cover of $Y_{n}$. Let $B_{i}=\left\{\left(x_{0}, x_{1}, \cdots, x_{i}, \cdots, x_{n}\right) \in Y_{n} \mid x_{i} \in\left[0, \Omega_{i}\right), x_{j}=\Omega_{j}\right.$ for $\left.j \neq i\right\}$. Then some element of $\mathscr{Q}$ must contain a cofinal subset of $B_{i}$.

Proof. Suppose not. Since $B_{i}$ is well-ordered by its $i$ th coordinate, then for each $j, 1 \leqq j \leqq k$, there exists an $\alpha_{i_{j}} \in\left[0, \Omega_{i}\right]$ such that $\left(\Omega_{0}, \Omega_{1}, \cdots, \alpha_{i_{j}}, \cdots, \Omega_{n}\right)$ is the first element of $B_{i}$ such that no element following it is in $U_{j}$. Let $\alpha=\max \left\{\alpha_{i_{j}}\right\}_{j=1}^{k}$. Then

$$
p=\left(\Omega_{0}, \Omega_{1}, \cdots, \beta_{i}, \cdots, \Omega_{n}\right)
$$

is not in any element of $\mathscr{C}$, if $\beta_{i}$ is the $i$ th coordinate of $p$, with $\beta_{i}>$ $\alpha$. This is a contradiction.

\section{Theorem 2.1. $Y_{1}$ is one dimensional.}

Proof. Let $\mathscr{Q}=\left\{U_{1}, U_{2}\right\}$ be a cover of $Y_{1}$ defined as follows: $U_{1}=Y_{1}-B_{0}$, where $B_{0}=\left\{\left(x, \Omega_{1}\right) \mid x \in\left[0, \Omega_{0}\right)\right\}, U_{2}=Y_{1}-B_{1}$, where $B_{1}=$ $\left\{\left(\Omega_{0}, x\right) \mid x \in\left[0, \Omega_{1}\right)\right\}$. We will show that any finite refinement $\mathscr{Y}$ of $\mathscr{C}$ must contain some pair of elements which intersect. We first note that some element of $\mathscr{V}$ must contain a cofinal subset of $B_{0}$, by Lemma 2.1. We assume that $V_{1}$ is such an element.

We show that $V_{1}$ must have a limit point in $B_{1}$. For each $\left(\alpha, \Omega_{1}\right) \in$ $V_{1} \cap B_{0}$, there exists $\beta_{\alpha}$ such that $(\alpha, \beta) \in V_{1}$ for $\beta \geqq \beta_{\alpha}$, since $V_{1}$ is an open set about $\left(\alpha, \Omega_{1}\right)$ and the set $\left\{(\alpha, x) \mid \alpha\right.$ fixed, $\left.x \in\left[0, \Omega_{1}\right]\right\}$ is homeomorphic to $X_{1}$. Then card $\left\{\beta_{\alpha} \mid\left(\alpha, \Omega_{1}\right) \in V_{1}\right\}<$ card $\Omega_{1}$, and therefore $\gamma=\sup \left\{\beta_{\alpha} \mid\left(\alpha, \Omega_{1}\right) \in V_{1}\right\}<\Omega_{1}$. Then $\left(\Omega_{0}, \gamma\right)$ is a limit point of $V_{1}$, but not in $U_{2}$ and therefore not in $V_{1}$. Thus any element of $\mathscr{V}$ containing $\left(\Omega_{0}, \gamma\right)$ must meet $V_{1}$. It follows that $Y_{1}$ is at least one dimensional.

We must now show that $Y_{1}$ is at most one dimensional. Let $\mathscr{C}$ be an arbitrary finite open cover of $Y_{1}$. We wish to find a finite refinement which is at most 1-dimensional-i.e. no three elements intersect. Recall that $Y_{1}=\left[0, \Omega_{0}\right] \times\left[0, \Omega_{1}\right]-\left\{\left(\Omega_{0}, \Omega_{1}\right)\right\}$.

Now $B_{0}=\left[0, \Omega_{0}\right) \times\left\{\Omega_{1}\right\}$ is a closed subset of $Y_{1}$ and is homeomorphic to the set of positive integers. Since $\mathscr{C}$ is a finite open cover of $Y_{1}$, we note, incidentally, that at least one element of $\mathscr{C}$ contains a cofinal subset of $B_{0}$. Let $U_{1}, U_{2}, \cdots, U_{n}$ be all the elements of $\mathscr{\mathscr { C }}$ which intersect $B_{0}$. If $V_{1}^{\prime}=U_{1} \cap B_{0}, V_{i}^{\prime}=\left(U_{i}-\bigcup_{j<i} U_{j}\right) \cap B_{0}$, then $\bigcup_{i=1}^{n} V_{i}^{\prime}=B_{0}$, and $V_{i}^{\prime}$ is open in $B_{0}$. For each point $\left(\alpha_{i_{\beta}}, \Omega_{1}\right) \in V_{i}^{\prime}$, there exists $\bar{\gamma}_{i_{\beta}} \in\left[0, \Omega_{1}\right)$ such that for all $\gamma \geqq \bar{\gamma}_{i_{\beta}},\left(\alpha_{i_{\beta}}, \gamma\right) \in U_{i}$. If $\bar{\gamma}_{i}=$ 
$\sup \left\{\bar{\gamma}_{i_{\beta}} \mid\left(\alpha_{i_{\beta}}, \Omega_{1}\right) \in V_{i}^{\prime}\right\}$, then $V_{i}^{\prime} \times\left[\bar{\gamma}_{i}, \Omega_{1}\right] \subseteq U_{i}$. If $\delta \geqq \max \left\{\bar{\gamma}_{i} \mid i=1, \cdots, n\right\}$, subject to the condition that $\delta$ is not a limit ordinal, then $V_{i}^{\prime} \times\left[\delta, \Omega_{1}\right] \subseteq$ $U_{i}, i=1, \cdots, n$. Let $W_{i}=V_{i}^{\prime} \times\left[\delta, \Omega_{1}\right]$, and we note that $W_{i}$ is an open subset of $u_{i}$. Now $\left[0, \Omega_{0}\right] \times[0, \delta-1]$ is a compact subset of $Y_{1}$, which is also open in $Y$. Therefore there exists a finite 0 -dimensional refinement $\mathscr{U}^{\prime}$ of $\mathscr{Z}$, covering this set. Now the collection of members of $\mathscr{C}^{\prime}$ together with the $\left\{W_{i}\right\}_{i=1}^{n}$, forms a 0 -dimensional finite open cover of $Y_{1}-\left(\left\{\Omega_{0}\right\} \times\left[\delta, \Omega_{1}\right)\right)$. We will add enough members to this collection in such a way that an at most 1-dimensional cover will result.

If $C=\left\{\Omega_{0}\right\} \times\left[\delta, \Omega_{1}\right)$, then a finite number of the elements of $\mathscr{C}$, say $U_{1}^{\prime \prime}, \cdots, U_{k}^{\prime \prime}$, cover $C$. Thus if $D_{i}=U_{i}^{\prime \prime} \cap C$, then $\left\{D_{i}\right\}_{i=1}^{k}$ is a finite open cover of $C$ in $C$. Since $C$ is 0 -dimensional, there exists a finite 0 -dimensional refinement, say $E_{1} \cdots E_{s}$. Now $\left[0, \Omega_{0}\right] \times E_{i}$ is open in $Y_{1}$. If $N_{i}=\left(\left[0, \Omega_{0}\right] \times E_{i}\right) \cap U_{E_{i}}$, where $U_{E_{i}}$ is any element of $\left\{U_{i}\right\}_{i=1}^{n}$ which contains $E_{i}$, then the collection $\left\{N_{i}\right\}_{i=1}^{s}$ is 0-dimensional collection.

Let $Q$ be the finite collection of open sets consisting of the members of $\mathscr{U}^{\prime}$, the members of $\left\{W_{i}\right\}_{i=1}^{n}$, and the members of $\left\{N_{i}\right\}_{i=1}^{s}$. Then $\mathscr{Q}$ is an at most 1-dimensional cover of $Y_{1}$, for the only possible intersections are between an $N_{i}$ and either an element of $\mathscr{U}^{\prime}$ or of $\left\{W_{i}\right\}_{i=1}^{n}$.

It follows that $Y_{1}$ is 1-dimensional.

\section{THEOREM 2.2. $\quad Y_{n}$ has dimension $\geqq n$.}

Proof. The proof is by induction on the method of proof of Theorem 2.1. Let $\mathscr{C}_{\ell}: U_{0}, U_{1}, \cdots, U_{n}$ be an open cover of $Y_{n}$ defined by $U_{i}=Y_{n}-\bigcup_{j \neq i} B_{n, j}$, where $B_{n, j}=\left\{\left(x_{0}, x_{1}, \cdots, x_{j}, \cdots, x_{n}\right) \in Y_{n} \mid x_{j} \in\right.$ $\left[0, \Omega_{j}\right)$ and $x_{k}=\Omega_{k}$ for $\left.k \neq j\right\}$. Let $\mathscr{V}$ be any finite refinement of $\mathscr{Z}$. By Lemma 2.1, for each $i, 0 \leqq i \leqq n$, there exists $V_{i} \in \mathscr{Y}$ such that $V_{i}$ contains a cofinal subset of $B_{n, i}$. We will show that $\bigcap_{i=0}^{n} V_{i} \neq \varnothing$, by showing that $\bigcap_{i=0}^{n-1} V_{i}$ has an end segment of $B_{n, n}$ as limit points. We note that $Y_{n-1}$ is homeomorphic to

$$
\left\{\left(x_{0}, x_{1}, \cdots, x_{n-1}, \Omega_{n}\right) \in Y_{n} \mid\left(x_{0}, x_{1}, \cdots, x_{n-1}\right) \in Y_{n-1}\right\} \subseteq Y_{n},
$$

and that $B_{n-1, i}$ is homeomorphic to $B_{n, i}$ for $0 \leqq i \leqq n-1$. We identify these in the remainder of the proof, without explicitly referring to the homeomorphism between them.

We assume, as an inductive hypothesis, that for any collection of $V_{i}$ 's chosen as above, $\bigcap_{i=0}^{n-1} V_{i} \neq \varnothing$. For $n=2$, this is proved in Theorem 2.1. Let $V_{i}^{\prime}=Y_{n-1} \cap V_{i}$, let $V^{\prime}=\bigcap_{i=0}^{n-1} V_{i}^{\prime}$, and let $V=\bigcap_{i=0}^{n-1} V_{i}$. 
Now card $Y_{n-1}<\operatorname{card} \Omega_{n}$, and therefore card $V^{\prime}<\operatorname{card} \Omega_{n}$. For $x \in$ $V^{\prime}$, let $\alpha_{x} \in\left[0, \Omega_{n}\right)$ be an ordinal such that if $\alpha>\alpha_{x}$, then $(x, \alpha) \in V$. We know that $\alpha_{x}$ exists since $V$ is open in $Y_{n}$ containing the point $\left(x, \Omega_{n}\right)$. Card $\left\{\alpha_{x} \mid x \in V^{\prime}\right\}<\operatorname{card} \Omega_{n}$. Therefore if $\bar{\alpha}=\sup \left\{\alpha_{x}\right\}$, then $\bar{\alpha}<\Omega_{n}$ and $V^{\prime} \times\left[\bar{\alpha}, \Omega_{n}\right] \subseteq V$.

Now let $y=\left(\Omega_{0}, \Omega_{1}, \cdots, \Omega_{n-1}, \beta\right) \in B_{n, n}$ such that $\beta \geqq \bar{\alpha}$. We wish to show that $y$ is a limit point of $V$. We accomplish this by showing that if $\left(\beta_{0}, \beta_{1}, \cdots, \beta_{n-1}, \beta\right) \in Y_{n}, \beta_{i}<\Omega_{i}$, then there exist $\gamma_{0}, \gamma_{1}, \cdots, \gamma_{n-1}$ such that $\gamma_{i}>\beta_{i}$ and $\left(\gamma_{0}, \gamma_{1}, \cdots, \gamma_{n-1}, \beta\right) \in V$. To this end, let $\eta_{i}$ be a non-limit ordinal $>\beta_{i}$ such that $\eta_{i} \in V_{i} \cap B_{n, i}$, let

$$
W_{i}=\left(\left[\eta_{i}, \Omega_{i}\right] \times \prod_{\substack{j=0 \\ j \neq i}}^{n} x_{j}\right) \cap V_{i} \text { for }
$$

$0 \leqq i \leqq n-1$, and let $W_{i}^{\prime}=W_{i} \cap Y_{n-1}$. Then $W_{i}^{\prime}$ is a neighborhood of a cofinal set in $B_{n, i} \subseteq Y_{n-1}$, and therefore, by the inductive hypothesis, $W_{i}^{\prime}=\bigcap_{i=0}^{n-1} W_{i}^{\prime} \neq \varnothing$. We note that $W^{\prime} \cong V^{\prime}$, and therefore there exists a point

$$
\left(\gamma_{0}, \gamma_{1}, \cdots, \gamma_{n-1}\right) \in W^{\prime} \subseteq V^{\prime}
$$

Then $\left(\gamma_{0}, \gamma_{1}, \cdots, \gamma_{n-1}, \beta\right) \in V^{\prime} \times\left[\bar{\alpha}, \Omega_{n}\right] \subseteq V$.

Thus we have shown that each point of $\left\{\left(\Omega_{0}, \Omega_{1}, \cdots, \Omega_{n-1}\right)\right\} \times\left[\bar{\alpha}, \Omega_{n}\right)$ is a limit point of $V$. Therefore any neighborhood $V_{n}$ of a cofinal set in $B_{n, n}$ must meet $V$ and $\bigcap_{i=0}^{n} V_{i} \neq \varnothing$. It follows that $\operatorname{dim} Y_{n} \geqq n$.

\section{THEOREM 2.3. $Y_{n}$ has dimension $\leqq n$.}

Proof. Let $\mathscr{C}$ be a finite open cover of $Y_{n}$. We wish to find a finite refinement of $\mathscr{C}$ which is an at most $n$-dimensional cover of $Y_{n}$. Let $B_{n, i}$ be defined as in the proof of Theorem 2.2, and again we don't distinguish in notation between $B_{n-1, i}$ in $Y_{n-1}$, and its homeomorphic copy $B_{n, i}$ in $Y_{n}$ for $1 \leqq i \leqq n-1$. We also identify $Y_{i}$ with $\left(Y_{i} \times \prod_{j=i+1}^{n}\left\{\Omega_{j}\right\}\right)$ in $Y_{n}$.

We assume, as an inductive hypothesis, that $Y_{n-1}$ has an at most $(n-1)$ dimensional refinement of any given cover. Let $\mathscr{Z}^{\prime}$ be the elements of $\mathscr{W}$ intersected with $Y_{n-1}$. Then $\mathscr{C}^{\prime}$ is a cover of $Y_{n-1}$, and by hypothesis, has an at most $(n-1)$-dimensional refinement. Let $\mathscr{V}^{\prime}: V_{1}^{\prime}, \cdots, V_{k}^{\prime}$ be such a refinement of $\mathscr{U}^{\prime}$. Let $V_{i}^{\prime} \subseteq U_{i}^{\prime}$. Note that it is possible that $U_{i}^{\prime}=U_{j}^{\prime}$ for $i \neq j$. Note also that card $Y_{n-1}<$ card $\Omega_{n}$. Fix $i$. For each $x \in V_{i}^{\prime}$, there exists $\alpha_{x_{i}}$ such that for $\alpha>$ $\alpha_{x_{i}},(x, \alpha) \in U_{i}$. Let $\bar{\alpha}_{\imath}=\sup \left\{\alpha_{x_{i}} \mid x \in V_{i}^{\prime}\right\}$ and we see that $\bar{\alpha}_{i}<\Omega_{n}$. Let $\bar{\alpha} \geqq \sup \left\{\bar{\alpha}_{i} \mid i=1, \cdots, k\right\}$ such that $\bar{\alpha}$ is a non-limit ordinal. Then $V_{i}=V_{i}^{\prime} \times\left[\bar{\alpha}, \Omega_{n}\right] \leqq U_{i} \in \mathscr{Z}_{L}$, for each $i, 1 \leqq i \leqq k$. Thus $Y_{n-1} \times\left[\bar{\alpha}, \Omega_{n}\right]$ is a subset of $Y_{n}$ which is covered by an at most $(n-1)$-dimen- 
sional finite collection, $\mathscr{V}: V_{1}, \cdots, V_{k}$, which refines $\mathscr{C}$. The points of $\left[B_{n, n} \cup\left(Y_{n-1} \times[0, \bar{\alpha})\right)\right]$ are the only points not yet covered. Now $Z_{n-1} \times[0, \bar{\alpha})$ is a zero dimensional clopen subset of $Y_{n}$, and therefore there exists a finite, zero dimensional refinement of $\mathscr{U}$ covering it, say $Q$.

It remains to cover the set of points of $\left\{\left(\Omega_{0}, \Omega_{1}, \cdots, \Omega_{n-1}\right)\right\} \times\left[\bar{\alpha}, \Omega_{n}\right)$. Call this set $A$. The elements of $\mathscr{C}$ intersected with $A$ is a cover of $A$, say $\mathscr{C}^{\prime \prime}$, and $A$ is zero dimensional. Thus there exists a zero dimensional finite refinement of $\mathscr{U}^{\prime \prime}$ which covers $A$, say $\mathscr{W}: W_{1}$, $W_{2}, \cdots, W_{s}$. Now fix $i$. For each $x \in W_{i} \leqq U_{i}$, there exists a clopen product neighborhood, $H_{i_{x}}=H_{0} \times H_{1} \cdots \times H_{n-1} \times W_{i_{x}}$, where $W_{i_{x}} \subseteq$ $W_{i}$ and such that $H_{i_{x}} \leqq U_{i}$. Let $H_{i}^{\prime}=\bigcup_{y \in W_{i}} H_{i_{y}}$. Then $x \in H_{i}^{\prime}, H_{i}^{\prime} \leqq$ $U_{i}$, and $H_{i}^{\prime}$ is open in $Y_{n}$. Further $H_{i}^{\prime} \cap H_{j}^{\prime}=\varnothing$ for $i \neq j$, and $H_{i}^{\prime} \cap$ $O_{j}=\varnothing$ for any $O_{j} \in \mathbb{Q}$. Let $\mathscr{C}=\left\{H_{1}^{\prime}, H_{2}^{\prime}, \cdots, H_{s}^{\prime}\right\}$. Then since at most $n$ elements of $\mathscr{V}$ have $\neq \varnothing$ intersection, the addition of $\mathscr{C l}$ to $\mathscr{V}$ will make at most $n+1$ elements intersect. Thus the cover defined by the elements of $\mathscr{Y}, \mathbb{Q}$, and $\mathscr{C l}$ is an at most $n$-dimensional cover of $Y_{n}$.

It follows that $Y_{n}$ is at most $n$-dimensional.

Theorem 2.4. $Y_{n}$ has dimension $n$.

Proof. Clear from Theorem 2.2 and 2.3.

THEOREM 2.5. $Y$ is infinite dimensional and contains subsets of dimension $n$, for every positive integer $n$.

Proof. It is clear that $Y$ contains $n$-dimensional subsets for all $n$, since $Y_{n}$ is homeomorphic to $\left\{\left(x_{0}, x_{1}, \cdots, x_{n}, \cdots\right) \in Y \mid x_{i}=\Omega_{i}\right.$ for all $i>n\} \subseteq Y$. We identify this set with $Y_{n}$ in our notation. Since $Y_{n}$ is closed in $Y$, it follows that $\operatorname{dim} Y=\infty$.

Theorem 2.6. $X$ is a 0-dimensional, compact, Hausdroff space, and contains subsets of dimension $n$, for $1 \leqq n \leqq \infty$.

Proof. By Theorem 2.5, $Y$ is $\infty$-dimensional and contains subsets of dimension $n$ for $1 \leqq n \leqq \infty$. But $Y \leqq X$. The theorem follows.

Corollary 2.6.1. For each $n, 1 \leqq n \leqq \infty$, there exists an $n$-dimensional continuum with subsets of dimension $k, 1 \leqq k \leqq \infty$.

Proof. $X_{i}$ is a totally ordered, totally disconnected compact Hausdorff space, and can be imbedded in a generalized arc, $A_{i}$, preserving the order of $X_{i}$, in such a way that if $x<y$ in $X_{i}$ and there does not exist $z$ in $X_{i}$ with $x<z<y$, then $A_{i}$ contains a real arc 
from $x$ to $y$.

Now $X=\prod_{j=0}^{\infty} X_{i}$ and is a compact totally disconnected Hausdorff space with a lattice-like structure, intuitively like the ordered $\infty$ tuples of integers in $R^{\infty}$. We fill in the lattice structure, to make a 1-dimensional grid, in the following way. Let $M=\prod_{i=1}^{\infty} A_{i}$ and let $M_{1}$ be the subset of $M$ defined by $M_{1}=\left\{\left(\alpha_{1}, \alpha_{2}, \cdots, \alpha_{i}, \cdots\right) \in M \mid\right.$ there exists at most one coordinate $\alpha_{k}$ such that $\left.\alpha_{k} \in A_{k}-X_{k}\right\}$. We show that $\operatorname{dim} M_{1}=1$. Clearly ind $M_{1}=1$, since, if $x \in M_{1}$ and $U$ is open with $x \in U$, then there exists a neighborhood $V$ of $x$ such that $V \cong$ $U$, and $B d V$ is homeomorphic to a subset of $\prod_{i=0}^{\infty} X_{i}$. Thus $B d V$ is 0 -dimensional, and ind $M_{1} \leqq 1$. But $M_{1}$ contains arcs. Thus ind $M_{1}=$ 1. Now, by Theorem B page 198 of [10], since $M_{1}$ is compact Hausdorff, $\operatorname{dim} M_{1} \leqq$ ind $M_{1}$. It follows that $\operatorname{dim} M_{1}=1$, since clearly $\operatorname{dim} M_{1}>0$.

Let $I_{i}=[0,1]$ and let $M_{n}=M_{1} \times \prod_{i=1}^{n-1} I_{i}$. By Theorem 4.7 of [2] the product of an $n$-dimensional compact space with the unit interval has dimension $\leqq n+1$. Now $M_{1}$ contains an interval, so that $\operatorname{dim} M_{2}=2$, and inductively, $\operatorname{dim} M_{n}=n$. Since $X \subseteq M_{n}$, the corollary follows.

3. On compactifications of $Y_{n}$ and $Y$. In [4, pg. 124], it is shown that the one point compactification of $Y_{1}=\beta Y_{1}=Z_{1}$. In this section, we show that the one points compactification of $Y_{n}=\beta Y_{n}=$ $Z_{n}$, for $1 \leqq n<\infty$. Thus for each $n, 1 \leqq n<\infty$, we have an example of a space of covering dimension $n$, whose Stone-Čech compactification is of dimension 0 . On pages $244-245$ of [4], it is proved that $\operatorname{dim} X=\operatorname{dim} \beta X$, if $X$ is normal. Thus we see the necessity of the hypothesis of normality. We note that in [4, pg. 248], it is pointed out that $Y_{1}$ is a space of positive dimension whose Stone-Čech compactification has dimension 0 .

Lemma 3.1. Let $f$ be continuous from $X_{n}$ (or $X_{n}-\left\{\Omega_{n}\right\}$ ) to the reals, $n \geqq 1$. Then there exists an end segment $E=\left[\alpha, \Omega_{n}\right)\left(\operatorname{or}\left[\alpha, \Omega_{n}\right]\right)$ such that $f$ is constant on $E$.

Proof. This is proved for $X_{1}$ on page 75 of [4]. It is clear that the same proof works for $X_{n}$.

THEOREM 3.1. The Stone-Čech compactification of $Y_{n}$ is $Z_{n}, n \geqq 1$.

Proof. For $n=1$, this is proved on page 124 of [4].

In general, $Y_{n}=\prod_{i=0}^{n} X_{i}-\left\{\left(\Omega_{0}, \Omega_{1}, \cdots, \Omega_{n}\right)\right\}$. By Theorems 6.4, 6.5 of [4] it is sufficient to show that every (bounded) continuous 
function from $Y_{n}$ to the reals, has a continuous bounded extension to $Z_{n}$. It follows from the argument below that any continuous real function on $Y_{n}$ is necessarily bounded.

We assume, as an inductive hypothesis, that every real continuous function on $Y_{n-1}$ can be extended to $Z_{n-1}$. Let $f: Y_{n} \rightarrow R$ be continuous. For each $x \in Y_{n-1}, W_{x}=\left\{(x, \alpha) \mid \alpha \in X_{n}\right\}$ is homeomorphic to $X_{n}$, and therefore $f \mid W_{x}$ is constant on an end segment, by Lemma 3.1; that is, there exists $\alpha_{x}$ such that $f(x, \alpha)=f\left(x, \alpha_{x}\right)$ for all $\alpha \geqq \alpha_{x}$. Further, if $W=\left\{\left(\Omega_{0}, \Omega_{1}, \cdots, \Omega_{n-1}, \alpha\right) \mid \alpha \in\left[0, \Omega_{n}\right)\right]$, then $f$ is constant on an end segment of $W$, also, say for $\alpha \geqq \alpha_{0}$. Now card $Z_{n-1}<\operatorname{card} \Omega_{n}$. Let $A=\left[\left\{\alpha_{0}\right\} \cup\left\{\alpha_{x} \mid x \in Y_{n-1}\right\}\right]$, and it follows that card $A<\operatorname{card} \Omega_{n}$. Therefore $\sup A<\Omega_{n}$. Let $\bar{\alpha} \geqq \sup A$ such that $\bar{\alpha}$ is a non-limit ordinal. Then $\left[Z_{n-1} \times\left(\bar{\alpha}, \Omega_{n}\right]\right]-\left\{\left(\Omega_{0}, \Omega_{1}, \cdots, \Omega_{n}\right)\right\}$ has the property that on each $\{x\} \times\left(\bar{\alpha}, \Omega_{n}\right], f$ is constant. It follows that for $x \in Y_{n-1}, f\left(\left(x, \Omega_{n}\right)\right)=$ $f((x, \beta))$, for each $\beta \geqq \bar{\alpha}$. Now, since $f \mid Y_{n-1} \times\left\{\Omega_{n}\right\}$ can be extended to $\left(\Omega_{0}, \cdots, \Omega_{n-1}, \Omega_{n}\right)$, say it has value $k$, then since this extension is unique, it has same value as $f$ on $\left(\Omega_{0}, \cdots, \Omega_{n-1}, \beta\right)$ for each $\beta \geqq \bar{\alpha}$. Thus we can extend $f$ to $Z_{n}$, by defining $f\left(\left(\Omega_{0}, \cdots, \Omega_{n}\right)\right)=k$.

It follows that $Z_{n}$ is the Stone-Cech compactification of $Y_{n}$.

\section{QUESTION. Is $X$ the Stone-Čech compactification of $Y$ ?}

Theorem 3.2. For each $n, 1 \leqq n<\infty$, there exists a (non-normal) space of dimension $n$, whose Stone-Čech compactification has dimen$\operatorname{sion} 0$.

Proof. Our spaces $Y_{n}$ satisfy the conditions of the theorem, by Theorems 2.4, 2.5, and 3.1.

4. The non-monotony of large inductive dimension. In $\S 2$, we proved that $\operatorname{dim} Y_{n}=n, 1 \leqq n \leqq \infty$. In this section, we show that Ind $Y_{n} \geqq n, 1<n<\infty$, Ind $Y_{1}=1$, and Ind $Y_{\infty}=\infty$. Thus Ind $X=$ 0 , but $X$ contains subsets $Y_{n}$ whose large inductive dimension is at least $n, 1 \leqq n \leqq \infty$.

Lemma 4.1. If $F_{1}$ and $F_{2}$ are disjoint closed sets in $\left[0, \Omega_{n}\right), 0<$ $n<\infty$, then one of them is bounded.

Proof. (See 5.12 (b) p. 74 of [4]. Proof is included here for completeness.) Suppose neither is bounded. Let $\left\{\alpha_{i}\right\}_{i=1}^{\infty}$ be a sequence of elements of $\left[0, \Omega_{n}\right)$ chosen inductively so that (1) $\alpha_{i}<\alpha_{i+1}$, (2) $\alpha_{i} \in$ $F_{1}$ for $i$ odd, and (3) $\alpha_{i} \in F_{2}$ for $i$ even. If $\gamma=\operatorname{lub}\left\{\alpha_{i}\right\}$ then $\gamma<\Omega_{n}$ and $\gamma$ is a limit point of both $F_{1}$ and $F_{2}$. Therefore $\gamma \in F_{1} \cap F_{2}$, and this is a contradiction. 
Lemma 4.2. Ind $\left[0, \Omega_{n}\right)=0,0 \leqq n<\infty$.

Proof. Case 1. $n>0$. Let $F$ be closed in $\left[0, \Omega_{n}\right)$, and let $U$ be a neighborhood of $F$. We wish to find an open set $V$ such that $F \cong V \subseteq U$ and $\mathrm{Bd} V=\varnothing$.

Suppose $F$ is bounded, there exists a clopen cover of $F$ by subsets $V_{\alpha}$ of $U$. Since $F$ is bounded then it must be compact, and therefore there exist $V_{\alpha_{1}}, \cdots, V_{\alpha_{k}}$ contained in $U$ such that $F \subseteq V=$ $\bigcup_{i=1}^{k} V_{\alpha_{i}}$. Then $V$ is open, $F \leqq V \leqq U$, and $\mathrm{Bd} V=\varnothing$.

If $F$ is not bounded then, by Lemma 4.1, $C(U)$ is bounded, and by a similar argument, we find the required set $V$.

Thus, in either event, there exists an open set $V$ such that $F \cong$ $V \subseteq U$ and $\operatorname{Bd} V=\varnothing$.

Case 2. $n=0$. Clear, since the nonnegative integers are discrete.

Lemma 4.3. If $\mathscr{C}$ is a finite open cover of $\left[0, \Omega_{n}\right), 1 \leqq n<\infty$, then some element of $\mathscr{Q}$ contains an end segment of $\left[0, \Omega_{n}\right)$.

Proof. Suppose no element of $\mathscr{U}$ contains an end segment. Then at least two elements of $\mathscr{C}$ must contain disjoint cofinal sets in $\left[0, \Omega_{n}\right)$. Let $U_{1}, \cdots, U_{k}$ be those elements of $\mathscr{U}$ which contain pairwise disjoint cofinal sets $A_{1}, \cdots, A_{k}$ respectively, in $\left[0, \Omega_{n}\right)$ with $A_{i} \cap U_{j}=\varnothing, j \neq i, i, j=1, \cdots, k$, and let $a_{\mathrm{i}, 1}, a_{2,1}, \cdots, a_{k, 1}$ be elements of $A_{1} \cdots, A_{k}$ respectively, such that $a_{i, 1}>x$ for all $x \notin \mathbf{U}_{j=1}^{k} U_{j}$. Let $b_{1} \in\left[0, \Omega_{n}\right)$ such that $b_{1}>a_{\imath, 1}, 1 \leqq i \leqq k$, let $a_{1,2}, a_{2,2}, a_{3,2}, \cdots, a_{k, 2}$ elements of $A_{1}, \cdots, A_{k}$ respectively, such that $a_{i, 2}>b_{1}$, and let $b_{2} \in\left[0, \Omega_{n}\right)$ such that $b_{2}>a_{i, 2}, 1 \leqq i \leqq k$.

Continue the above process inductively, and let $b=\sup \left\{b_{i}\right\}$. Then $b<\Omega_{n}$ and $b$ is a limit point of $A_{i}, 1 \leqq i \leqq k$. Further, if $b \in U_{j}$ then $j \in\{1,2, \cdots, k\}$ and $U_{j}$ contains points of each $A_{i}, 1 \leqq i \leqq k$. But $A_{i} \cap U_{j}=\varnothing$ for $i \neq j$ when $1 \leqq i \leqq k$, and this is a contradiction.

Lemma 4.4. If $F_{1}$ and $F_{2}$ are disjoint closed sets in $\prod_{i=0}^{n-1}\left[0, \Omega_{i}\right] \times$ $\left[0, \Omega_{n}\right)$, then at least one of $F_{1}$ and $F_{2}$ is bounded away from the corner point of $Y_{n}$ in the $n^{\text {th }}$ direction; that is, there exist non-limit ordinals $\alpha_{0}, \alpha_{1} \cdots, \alpha_{n}$ such that $\prod_{i=0}^{n-1}\left[\alpha_{i}, \Omega_{i}\right] \times\left[\alpha_{n}, \Omega_{n}\right)$ misses, say, $F_{2}$. (The corner point of $Y_{n}$ is $\left(\Omega_{0}, \cdots, \Omega_{n}\right)$ even though this point does not belong to $Y_{n}$.)

Proof. Let $B_{n}=\left\{\left(\Omega_{0}, \cdots, \Omega_{n-1}, x\right) \mid x \in\left[0, \Omega_{n}\right)\right\}$ and we see by Lemma 4.1, not both $F_{1} \cap B_{n}$ and $F_{2} \cap B_{n}$ can be unbounded in $B_{n}$. Thus we assume that there exists a non-limit ordinal $\alpha_{n}$ such that if $\alpha \geqq \alpha_{n}$ then $\left(\Omega_{0}, \Omega_{1}, \cdots, \Omega_{n-1}, \alpha\right) \notin F_{2}$. 
Now, suppose the lemma is false. Then for each point $\left(\beta_{0}, \beta_{1}, \cdots\right.$, $\left.\beta_{n}\right)$ with $\beta_{n}>\alpha_{n}$, and $\beta_{i}<\Omega_{i}$ for all $i$, there exists another point $\left(\beta_{0}^{\prime}, \beta_{1}^{\prime}, \cdots, \beta_{n}^{\prime}\right)$ in $F_{2}$ such that $\beta_{i}<\beta_{i}^{\prime} \leqq \Omega_{i}$ for $0 \leqq i \leqq n-1$ and $\beta_{n}<$ $\beta_{n}^{\prime}<\Omega_{n}$. Thus after a countable number of choices, with each point closer, in at least one coordinate to $\left(\Omega_{0}, \cdots, \Omega_{n}\right)$ than the preceding point (closer in $0^{\text {th }}$ coordinate whenever possible), we exhaust all possibilities for $\beta_{0}$. This is true because there are only a countable number of points in $X_{0}$. Then if $\beta_{i, 1}$ denotes $\sup \left\{\beta_{i}^{\prime} \mid\right.$ for each choice of $\left.\beta_{0}^{\prime} \leqq \Omega_{0}\right\}$ it follows that $\left(\Omega_{0}, \beta_{1,1}, \beta_{2,1}, \cdots, \beta_{n, 1}\right)$ is a point or limit point of $F_{2}$ and therefore must be in $F_{2}$. Further, each $\beta_{i, 1} \leqq \Omega_{i}$, $1 \leqq i<n$, and $\beta_{n, 1}<\Omega_{n}$.

We continue the process inductively and note that after at most $\left|\Omega_{1}\right|$ choices, we obtain a point $\left(\Omega_{0}, \Omega_{1}, \beta_{2,2} \cdots, \beta_{n, 2}\right)$ as a point or limit point of $F_{2}$, and therefore in $F_{2}$, and such that $\beta_{i, 2} \leqq \Omega_{i}, 2 \leqq i<n$, with $\beta_{n, 2}<\Omega_{n}$. After $n$ steps, we obtain a point $\left(\Omega_{0}, \Omega_{1}, \cdots, \Omega_{n-1}, \beta_{n, n}\right)$ in $B_{n}$ as a point or limit point of $F_{2}$ and therefore in $F_{2}$. But $\beta_{n, n}>$ $\alpha_{n}$ and this is a contradiction. Thus $F_{2}$ must be bounded away from the corner point of $Y_{n}$ in the $n^{\text {th }}$ direction.

LEMmA 4.5. If $B \cong A$ and $U \subseteq A$, then $\operatorname{Bd}_{B}(U \cap B) \subseteq \operatorname{Bd}_{A}(U)$.

Proof. Let $x \in B d_{B}(U \cap B)$. Then each neighborhood of $x$ contains points of $U \cap B$ and of $C(U) \cap B$ and therefore contains points of both $U$ and $C(U)$. It follows that $x \in \operatorname{Bd}_{A}(U)$.

REMARK. The following two lemmas are well known among dimension theorists. However, we include them here since they may not have been explicitly stated in other published work. The proof is the same as the proof for the metric case [10, II. 1.A].

Lemma 4.6. If Ind $A=0$ and $B$ is a closed subset of $A$, then Ind $B=0$.

Lemma 4.7. If Ind $A=k$ and $B$ is a closed subset of $A$, then Ind $B \leqq k$.

REMARK. The next proposition is not used in the rest of the paper, but is included for its (possible) interest. Also, the proof of this proposition is referred to in Theorem 4.1.

Proposition 4.1. ${ }^{1}$ If $S=\left(\prod_{i=0}^{n-1} X_{i}\right) \times\left[0, \Omega_{n}\right)$, then $\operatorname{dim} S=\operatorname{Ind} S=0$.

1 The referee has pointed out that this theorem follows easily from a theorem of Morita (Topogical completeness and $M$-spaces, Sci. Reports Tokyo Kyoiku Daigaku. Sec. A 10 (1970) 271-288) that $\operatorname{dim} X \times Y \leqq \operatorname{dim} X+\operatorname{dim} Y$ if $X$ is paracompact, locally compact, and $Y$ is pseudoparacompact. 
Proof. Let $\mathscr{U}$ be a finite open cover of $S$. Each $\{x\} \times\left[0, \Omega_{n}\right)$ is homeomorphic to $\left[0, \Omega_{n}\right.$ ), and therefore, by Lemma 4.3 , some end segment of $\{x\} \times\left[0, \Omega_{n}\right)$, say $\{x\} \times\left[\alpha_{x}, \Omega_{n}\right)$, is a subset of every element of $\mathscr{U}$ which it meets. Since card $\left(\prod_{i=0}^{n-1} X_{i}\right)<\operatorname{card}\left[0, \Omega_{n}\right)$, let $\alpha^{\prime}=$ $\sup \left\{\alpha_{x} \mid x \in \prod_{i=0}^{n-1} X_{i}\right\}$ and let $\alpha>\alpha^{\prime}$ be a non-limit ordinal. Then for each $x$, there is a $j$ such that $\left(\{x\} \times\left[\alpha, \Omega_{n}\right)\right) \subseteq U_{j}$ Further, if $\{x\} \times$ $\left[\alpha, \Omega_{n}\right.$ ) meets several $U_{j}$ 's, then it is a subset of each of them. Consider $\left(\prod_{i=0}^{n-1} X_{i}\right) \times\{\alpha\}$. This is a compact zero dimensional space, and $\mathscr{U}^{\prime}=$ $\left\{U_{j} \cap\left(\prod_{i=0}^{n-1} X_{i} \times\{\alpha\}\right) \mid U_{j} \in \mathscr{U}\right\}$ is a finite open cover of this space. Thus there exists a zero dimensional refinement $\mathscr{V}^{\prime}$ of $\mathscr{C}^{\prime}$. For each $V^{\prime} \in \mathscr{V}^{\prime}, V^{\prime} \times\left[\alpha, \Omega_{n}\right) \subseteq$ some $U_{j}$, and the collection $\mathscr{V}=\left\{V^{\prime} \times\left[\alpha, \Omega_{n}\right) \mid V^{\prime} \in\right.$ $\left.\mathscr{V}^{\prime}\right\}$ is a zero dimensional cover of $\left(\prod_{i=0}^{n-1} X_{i}\right) \times\left[\alpha, \Omega_{n}\right)$ refining $\mathscr{U}$.

Now since $\prod_{i=0}^{n-1} X_{i} \times[0, \alpha-1]$ is a zero dimensional compact Hausdorff space, there exists a finite zero dimensional refinement $\mathscr{Y}$ of $\mathscr{C}$ covering this set. Then the collection $\mathscr{Y} \cup \mathscr{W}$ is a finite zero dimensional refinement of 26 . It follows that $\operatorname{dim} S=0$.

Now in the appendix of [7], it is shown that for any space $S$, $\operatorname{dim} S=0$ iff Ind $S=0$. Thus the lemma is proved.

Theorem 4.1. Ind $Y_{1}=1$.

Proof. If $B_{0}=\left\{\left(x, \Omega_{1}\right) \mid x \in\left[0, \Omega_{0}\right)\right\}, B_{1}=\left\{\left(\Omega_{0}, x\right) \mid x \in\left[0, \Omega_{1}\right)\right\}$, and $U=$ $Y_{1}-B_{1}$, then $B_{0}$ is a closed subset of $Y_{1}$ and $B_{0} \subseteq U$, with $U$ open in $Y_{1}$. As in the proof of Theorem 2.1, we see that for any open set $V$ such that $B_{0} \leqq V \leqq U, \mathrm{Bd} V$ must contain $a \neq \varnothing$ end segment of $B_{1}$. Thus Ind $Y_{1} \geqq 1$.

We now show Ind $Y_{1} \leqq 1$. Let $F$ be closed in $Y_{1}$ and let $U$ be and neighborhood of $F . \quad F$ and $\mathrm{Bd} U$ are disjoint closed sets in $Y_{1}$.

If $F$ contains a cofinal set in $B_{1}$, then by Lemma 4.1, $\mathrm{Bd} U$ misses an end segment of $B_{1}$ and by Lemma 4.4, there exist non-limit ordinals $\alpha_{0}$ and $\alpha_{1}$ such that $\left[\alpha_{0}, \Omega_{0}\right] \times\left[\alpha_{1}, \Omega_{1}\right)$ misses $\mathrm{Bd} U$. Thus $\mathrm{Bd} U \subseteq K \cup$ $\left(\left[\alpha_{0}, \Omega_{0}\right] \times\left\{\Omega_{1}\right\}\right)$, where $K$ is the totally disconnected compact open subset of $Y_{1}$ defined by $K=Z_{1}-\left[\alpha_{0}, \Omega_{0}\right] \times\left[\alpha_{1}, \Omega_{1}\right]$. Then $K \cap \mathrm{Bd} U$ can be covered by a finite number of clopen sets $V_{1}, \cdots, V_{s}$, each of which is a subset of $K$ and misses $F$, so that $V=U-\bigcup_{i=1}^{s} V_{i}$ is open, contains $F$, and $\mathrm{Bd} V=\varnothing$ or $\mathrm{Bd} V \subseteq\left[\alpha_{0}, \Omega_{0}\right] \times\left\{Q_{1}\right\}$.

If $F$ contains no cofinal set in $B_{1}$, then by an argument like that of the above paragraph, $F \subseteq L \cup\left(\left[\beta_{0}, \Omega_{0}\right) \times\left\{\Omega_{1}\right\}\right)$ where $L$ is a totally disconnected compact open subset of $Y_{1}$ defined by $L=Z_{1}-\left[\beta_{0}, \Omega_{0}\right] \times$ $\left[\beta_{1}, \Omega_{1}\right]$, and $\beta_{0}$ and $\beta_{1}$ are some non-limit ordinals. Then $L \cap F$ can be covered by a finite number of clopen sets $W_{1}, \cdots, W_{t}$, each of which is a subset of $U \cap L$, so that if $W=\bigcup_{i=1}^{t} W_{i}$, then $\mathrm{Bd} W=\varnothing$. From this we see that if $F \subseteq L$, there exists an open set $W$ such that 
$F \subseteq W \leqq U$ and $\mathrm{Bd} W=\varnothing$. Otherwise $F \cap\left(Y_{1}-L\right) \subseteq\left[\beta_{0}, \Omega_{0}\right] \times\left\{\Omega_{1}\right\}$. Let $F^{\prime \prime}=F \cap\left(Y_{1}-L\right)$ and let $U^{\prime}=U \cap\left(Y_{1}-L\right)$. It suffices to show that there exists $W^{\prime}$ open such that $F^{\prime} \subseteq W^{\prime} \subseteq U^{\prime}$ and $\mathrm{Bd} W^{\prime}$ is at most 0 -dimensional (in the large inductive sense).

To this end, we note that $U^{\prime}$ is a neighborhood of $F^{\prime}, F^{\prime} \cong$ $\left(\left[\beta_{0}, \Omega_{0}\right) \times\left\{\Omega_{1}\right\}\right)$ and, since there are only countably many elements in $F^{\prime}$, by the method used in Proposition 4.1, there exists a non-limit ordinal $\gamma$ such that if $W^{\prime}=\left(\left(F^{\prime \prime} \cap B_{0}\right) \times\left[\gamma, \Omega_{1}\right]\right)$, then $W^{\prime} \subseteq U^{\prime}$. Since $\left[0, \Omega_{0}\right)$ is discrete, $W^{\prime}$ is open, so that $W^{\prime}$ is open, contains $F^{\prime}$ and $\mathrm{Bd} W^{\prime} \subseteq B_{1}$ or $\mathrm{Bd} W^{\prime}=\varnothing$. Thus $\mathrm{Bd} W^{\prime}$ is at most zero dimensional. The theorem follows.

Corollary 4.1.1. If $A=\left[\alpha, \Omega_{0}\right] \times\left[\beta, \Omega_{1}\right]-\left\{\left(\Omega_{0}, \Omega_{1}\right)\right\}$ then Ind $A=1$.

Proof. Clear from the proof of Theorem 4.1.

CoROllary 4.1.2. Let $Y_{1}^{\prime}=\left[0, \Omega_{1}\right] \times\left[0, \Omega_{2}\right]-\left\{\left(\Omega_{1}, \Omega_{2}\right)\right\}$ and let $Y_{1}^{\prime}(\alpha, \beta)=\left[\alpha, \Omega_{1}\right] \times\left[\beta, \Omega_{2}\right]-\left(\Omega_{1}, \Omega_{2}\right)$. Then Ind $Y_{1}^{\prime}=1$ and Ind $Y_{1}^{\prime}(\alpha, \beta)=1$.

Proof. A simple modification of the proof of Theorem 4.1 shows this.

Definition. Let $Y_{n-1}^{\prime}=\prod_{i=1}^{n}\left[0, \Omega_{i}\right]-\left\{\left(\Omega_{1}, \Omega_{2}, \cdots, \Omega_{n}\right)\right\}$. By a corner of $Y_{n-1}^{\prime}$ we mean a set of the form $\prod_{r=1}^{n}\left[\alpha_{i}, \Omega_{i}\right]-\left\{\left(\Omega_{1}, \cdots, \Omega_{n}\right)\right\}$, where $\alpha_{i}$ is a non-limit ordinal, $i=1, \cdots, n$. In the proof below, we identify $Y_{n-1}^{\prime}$ with the set of points of $Y_{n}$ whose first coordinate is $\Omega_{0}$.

THEOREM 4.2. Ind $Y_{n} \geqq n, 1 \leqq n<\infty$.

Proof. If $B_{0}=\left\{\left(x, \Omega_{1}, \Omega_{2}, \cdots, \Omega_{n}\right) \in Y_{n} \mid x \in\left[0, \Omega_{0}\right)\right\}$ and

$$
B_{i}=\left\{\left(\Omega_{0}, \cdots, \Omega_{i-1}, x, \Omega_{i+1}, \cdots, \Omega_{n}\right) \in Y_{n} \mid x \in\left[0, \Omega_{i}\right)\right\}
$$

then $B_{0}$ is closed and $U=Y_{n}-Y_{n-1}^{\prime}$ is a neighborhood of $B_{0}$. We assume as an inductive hypothesis that any corner of $Y_{n-1}^{\prime}$ has large inductive dimension at least $n-1$, and note that for $n=2$, this is just Corollary 4.1.2. We will show that any neighborhood $V$ of $B_{0}$ such that $V \cong U$ must contain a corner of $Y_{n-1}^{\prime}$ on its boundary. As in the proofs of $\S 2$, we do not distinguish in notation between $Y_{n-1}^{\prime}$ and its copy in $Y_{n}$.

To this end, we first prove that if $V$ is any neighborhood of $B_{0}$ such that $V \subseteq U$, then there exist non-limit ordinals $\alpha_{1}, \alpha_{2}, \cdots, \alpha_{n}$ such that $B_{0} \times \prod_{i=1}^{n}\left[\alpha_{i}, \Omega_{i}\right] \subseteq V$. By an analogous argument to the proof of Theorem 2.1, there is a non-limit ordinal $\alpha_{1}$ such that $B_{0} \times$ 
$\left[\alpha_{1}, \Omega_{1}\right] \subseteq U$. Inductively assume that there exist non-limit ordinals $\alpha_{1}, \cdots, \alpha_{k-1}$ such that $B_{0} \times \prod_{i=1}^{k-1}\left[\alpha_{i}, \Omega_{i}\right] \subseteq V$. Now each point of $B_{0} \times$ $\prod_{i=1}^{k-1}\left[\alpha_{i}, \Omega_{i}\right]$ is the last point of a "vertical set" over it in $Y_{n}$ with the vertical set homeomorphic to $\left[0, \Omega_{i}\right]$, and card $\left(B_{0} \times \prod_{i=1}^{k-1}\left[\alpha_{i}, \Omega_{i}\right]\right)$ $<\Omega_{k}$. Thus for each of these "vertical sets", there exists $\beta<\Omega_{k}$ such that the end segment below $\beta$ lies in $V$, since $V$ is a neighborhood of the last point of this segment. If we let $\alpha_{k}$ be greater than or equal to $\sup \left\{\beta \mid \beta\right.$ is obtained as described for each point of $B_{0} \times$ $\left.\prod_{i=1}^{k-1}\left[\alpha_{i_{k}}, \Omega_{i}\right]\right\}$ with $\alpha_{k}$ a non-limit ordinal, then $\alpha_{k}<\Omega_{k}$ and $B_{0} \times$ $\prod_{i=1}^{k}\left[\alpha_{i}, \Omega_{i}\right] \subseteq V$. Our assertion about the existence of $\alpha_{1}, \cdots, \alpha_{n}$ follows.

Thus we see that each point of

$$
A=\left[\left\{\Omega_{0}\right\} \times \prod_{i=1}^{n}\left[\alpha_{i}, \Omega_{i}\right]-\left\{\left(\Omega_{0}, \Omega_{1}, \cdots, \Omega_{n}\right)\right\}\right]
$$

is a limit point of $B_{0} \times \prod_{\imath=1}^{n}\left[\alpha_{i}, \Omega_{i}\right]$, so that $A \subseteq \mathrm{Bd} V$. But Ind $A=$ $n-1$, and it follows that any neighborhood $V$ of $B_{0}$ such that $V \cong$ $U$ has at least $(n-1)$ dimensional (Ind) set on its boundary. Thus Ind $Y_{n} \geqq n$.

Corollary 4.2.1. Let $T=\prod_{\substack{i=0 \\ i \neq i_{0}}}^{n}\left[0, \Omega_{i}\right]-\left\{\left(\Omega_{0}, \cdots, \hat{\Omega}_{i_{3}}, \cdots, \Omega_{n}\right)\right\}$ and let $T^{\prime}=\prod_{i=0}^{n}\left[\alpha_{i}, \Omega_{i}\right]-\left\{\left(\Omega_{0}, \cdots, \widehat{\Omega}_{i_{n}}, \cdots, \Omega_{n}\right)\right\}$. Then each of $T$ and $T^{\prime}$ has large inductive dimension at least $n-1$.

Proof. A modification of the proof of Theorem 4.2 works.

Theorem 4.3. Ind $Y=\infty$.

Proof. $Y_{n} \subseteq Y$ homeomorphically and we identify $Y_{n}$ with its image in $Y$. Since Ind $Y_{n} \geqq n$, and $Y_{n}$ is closed in $Y$, Ind $Y=\infty$ 。

THEOREM 4.4. $X$ is a totally disconnected compact Hausdorff space with Ind $X=0$, but $X$ contains subsets $Y_{n}, 1 \leqq n \leqq \infty$, with Ind $Y_{n} \geqq$ $n$; Ind $Y_{1}=1$, and Ind $Y_{\infty}=\infty$.

Proof. Follows from Theorems 4.2 and 4.3 .

QUESTION. Is Ind $Y_{n}=n$ for $1<n<\infty$ ?

\section{REFERENCES}

1. P. S. Aleksandrov, The present status of the theory of dimension, Amer. Math. Soc. Translations, Series 2, 1, (1955), 1-26. Original article in Russian (1951).

2. P. Alexandroff, On the dimension of normal spaces, Proc. Royal Soc. London, Series A, 189 (1947), 11-39. 
3. C. H. Dowker, Local dimension of normal spaces, Quart. J. of Math., Oxford (2) 6 (1955), 101-120.

4. Gillman and Jerison, Rings of Continuous Functions, Van Nostrand, Princeton, N. J., 1960.

5. P. R. Halmos, Naive Set Theory, Van Nostrand, Princeton, N. J., 1960.

6. D. W. Henderson, An in finite dimensional compactum with no positive dimensional compact subsets - a simpler construction, Amer. J. of Math., 89 (1967), 105-121.

7. W. Hurewicz and H. Wallman, Dimension Theory, Princeton Univ. Press, Princeton, New Jersey, 1948.

8. J. R. Isbell, Uniform Spaces, Amer. Math. Soc., Providence, Rhode Island, (1964), $102-103$.

9. J. L. Kelley, General Topology, Van Nostand, Princeton, N. J., 1955.

10. J. Nagata, Modern Dimension Theory, Interscience Publishers of John Wiley and Sons, New York, 1965.

Received February 4, 1971 and in revised form August 17, 1971. Presented to the American Mathematical Society meeting in Athens, Georgia, on Nov. 21, 1970. The author would like to express her appreciation to the referee for his kind comments and criticisms.

UNIVERSITY OF FLORIDA 


\title{
PACIFIC JOURNAL OF MATHEMATICS
}

\author{
EDITORS
}

H. SAMELSON

Stanford University

Stanford, California 94305

C. R. HOBBY

University of Washington

Seattle, Washington 98105

\section{J. DugundJI}

Department of Mathematics

University of Southern California

Los Angeles, California 90007

RICHARD ARENS

University of California

Los Angeles, California 90024

\section{ASSOCIATE EDITORS}
E. F. BeCKeNBACH
B. H. NeUmanN
F. WoLF
K. YoSHIDA

\section{SUPPORTING INSTITUTIONS}

\author{
UNIVERSITY OF BRITISH COLUMBIA \\ CALIFORNIA INSTITUTE OF TECHNOLOGY \\ UNIVERSITY OF CALIFORNIA \\ MONTANA STATE UNIVERSITY \\ UNIVERSITY OF NEVADA \\ NEW MEXICO STATE UNIVERSITY \\ OREGON STATE UNIVERSITY \\ UNIVERSITY OF OREGON \\ OSAKA UNIVERSITY
}

\author{
UNIVERSITY OF SOUTHERN CALIFORNIA \\ STANFORD UNIVERSITY \\ UNIVERSITY OF TOKYO \\ UNIVERSITY OF UTAH \\ WASHINGTON STATE UNIVERSITY \\ UNIVERSITY OF WASHINGTON

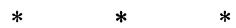 \\ AMERICAN MATHEMATICAL SOCIETY \\ NAVAL WEAPONS CENTER
}

The Supporting Institutions listed above contribute to the cost of publication of this Journal, but they are not owners or publishers and have no responsibility for its content or policies.

Mathematical papers intended for publication in the Pacific Journal of Mathematics should be in typed form or offset-reproduced, (not dittoed), double spaced with large margins. Underline Greek letters in red, German in green, and script in blue. The first paragraph or two must be capable of being used separately as a synopsis of the entire paper. The editorial "we" must not be used in the synopsis, and items of the bibliography should not be cited there unless absolutely necessary, in which case they must be identified by author and Journal, rather than by item number. Manuscripts, in duplicate if possible, may be sent to any one of the four editors. Please classify according to the scheme of Math. Rev. Index to Vol. 39. All other communications to the editors should be addressed to the managing editor, Richard Arens, University of California, Los Angeles, California, 90024.

50 reprints are provided free for each article; additional copies may be obtained at cost in multiples of 50 .

The Pacific Journal of Mathematics is published monthly. Effective with Volume 16 the price per volume (3 numbers) is $\$ 8.00$; single issues, $\$ 3.00$. Special price for current issues to individual faculty members of supporting institutions and to individual members of the American Mathematical Society: $\$ 4.00$ per volume; single issues $\$ 1.50$. Back numbers are available.

Subscriptions, orders for back numbers, and changes of address should be sent to Pacific Journal of Mathematics, 103 Highland Boulevard, Berkeley, California, 94708.

PUBLISHED BY PACIFIC JOURNAL OF MATHEMATICS, A NON-PROFIT CORPORATION

Printed at Kokusai Bunken Insatsusha (International Academic Printing Co., Ltd.), 270, 3-chome Totsuka-cho, Shinjuku-ku, Tokyo 160, Japan. 


\section{Pacific Journal of Mathematics}

\section{Vol. 41, No. $3 \quad$ BadMonth, 1972}

George E. Andrews, Two theorems of Gauss and allied identities proved arithmetically.................................. 563

Stefan Bergman, On pseudo-conformal mappings of circular domains . . . . 579

Beverly L. Brechner, On the non-monotony of dimension ............ 587

Richard Anthony Brualdi and John H. Mason, Transversal matroids and Hall's theorem .................................... 601

Philip Throop Church and James Timourian, Differentiable maps with

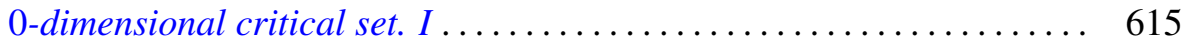

John H. E. Cohn, Squares in some recurrent sequences ............. 631

Robert S. Cunningham, Edgar Andrews Rutter and Darrell R. Turnidge, Rings of quotients of endomorphism rings of projective modules ......

Eldon Dyer and S. Eilenberg, An adjunction theorem for locally equiconnected spaces................................... 669

Michael W. Evans, On commutative P. P. rings................. 687

Ronald Lewis Graham, Hans Sylvain Witsenhausen and Hans Zassenhaus, On tightest packings in the Minkowski plane ..................

Stanley P. Gudder, Partial algebraic structures associated with

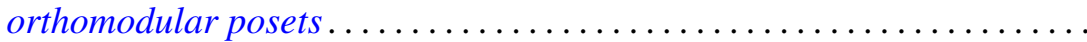

Karl Edwin Gustafson and Gunter Lumer, Multiplicative perturbation of

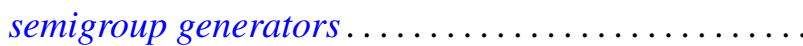

Kurt Kreith and Curtis Clyde Travis, Jr., Oscillation criteria for selfadjoint elliptic equations...

Lawrence Louis Larmore, Twisted cohomology theories and the single obstruction to lifting...........................

Jorge Martinez, Tensor products of partially ordered groups . . .

Robert Alan Morris, The inflation-restriction theorem for Amitsur cohomology ...

Leo Sario and Cecilia Wang, The class of $(p, q)$-biharmonic functions ...

Manda Butchi Suryanarayana, On multidimensional integral equations of

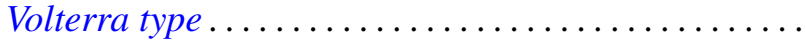

